\title{
GEORGIA, UKRAINE AND MOLDOVA, THE EU EASTERN PARTNERSHIP MEMBER STATES: MODERN CHALLENGES
}

\author{
RAMAZ PUTKARADZE \\ Doctor of Economics, Associate Professor \\ Ivane Javakhishvili Tbilisi State University, Georgia \\ ramaz.putkaradze@tsu.ge
}

Abstract. The study of various initiatives of the European Union is highly pressing in terms of current globalization and regional integration processes. The Eastern Partnership is an EU initiative aimed at deepening and strengthening relations between the EU, its member states and the six countries of its Eastern neighborhood. The study of the issues concerning the economic development of the three associated countries (Georgia, Ukraine and Moldova) of Eastern Partnership is very important. Therefore, if these countries carry out significant reforms and the EU supports the development of the initiative, the integration of all three countries with the EU will possibly deepen.

The paper discusses the indicators and peculiarities of socio-economic development of the EU Eastern Partnership Associated Countries. These countries are actively seeking for further integration into the global economy.

Trade relations between Georgia, Moldova and Ukraine and the European Union have significant prospects. The share of Georgian exports to the EU in total exports amounted to 22\%. EU plays an important role in Moldova's exports; in 2019 the share of the EU in Moldova's exports was $64 \%$, which is the highest among the associated countries. Ukraine's trade with the $\mathrm{EU}$ is also high among these countries.

In 2019, Georgia's foreign trade turnover (\%) accounted for $75 \%$ of GDP, which is quite high. Negative trade balance is a very important problem for the country; trade deficit (\%) accounted for 32\% of GDP. In 2019, the share of trade turnover (\%) of Moldova and Ukraine reached $72-72 \%$ of GDP.

It should be noted that territorial integrity of all these countries within internationally recognized borders is violated which significantly hinders the economic development of the country; in addition, it also poses a serious threat to maintaining security and stability; and hinders their striving towards the EU.

The Eastern Partnership allows its member countries, including Georgia, to accelerate the process of trade and economic integration with the European Union through bilateral and multilateral cooperation.

The EU Eastern Partnership initiative promotes further integration of the member states into the EU, as well as the socioeconomic development of its member states. The Eastern partner countries have a common communist past.

Georgia has the highest negative trade balance in foreign trade in goods among Eastern Partner countries. In addition, Georgia has the highest negative trade balance and negative import-export ratio. In case successful economic reforms are carried out Georgia might be the first Eastern Partner country to be awarded the status of an EU candidate country.

None of the Eastern Partner countries is a member of NATO. There is no direct link between the EU and NATO; however, the recent enlargements have shown that before joining the EU the country first becomes a member of NATO.

Georgia should continue further integration with the European Union and increase trade and economic cooperation with the EU and its member states. Increasing Georgia's exports to the EU remains a major challenge for the country. The Eastern Partner countries have to continue their effort to develop their economies and further integrate with the EU and the international community.

Thus, Georgia has a good opportunity to get closer to the EU, develop the national economy, make it competitive and increase trade and economic cooperation with the EU.

The Eastern Partnership allows its member countries, including Georgia, to accelerate the process of trade and economic integration with the European Union through bilateral and multilateral cooperation.

KEYWORDS: EASTERN PARTNERSHIP COUNTRIES, GEORGIA, MOLDOVA, UKRAINE, FOREIGN TRADE, GDP, ECONOMIC INTEGRATION.

For citation: Putkaradze, R. (2021). Georgia, Ukraine and Moldova, the EU Eastern Partnership Member States: Modern Challenges. Globalization and Business. 12, 66-73. (In Georgian). https://doi.org/10.35945/gb.2021.12.009 


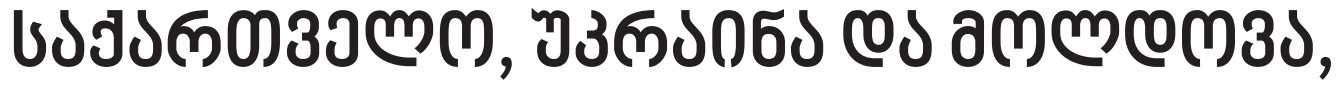

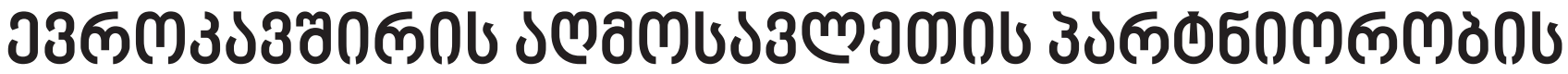

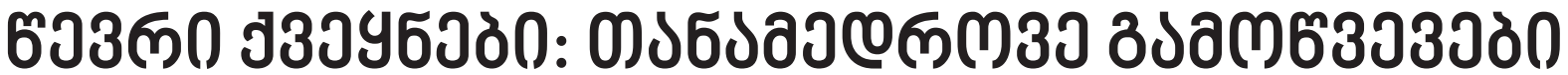

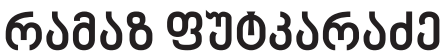

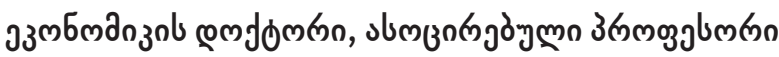

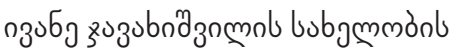

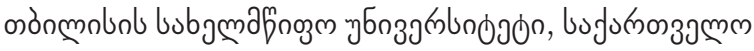

ramaz.putkaradze@tsu.ge

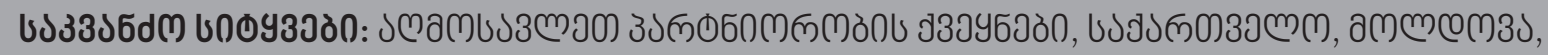

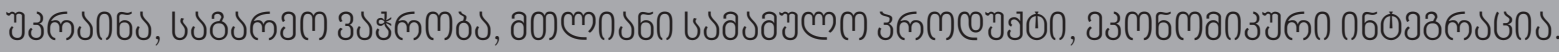

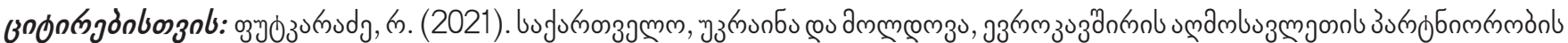

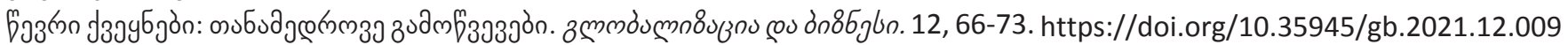

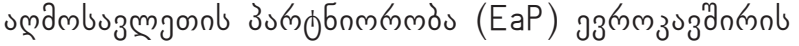

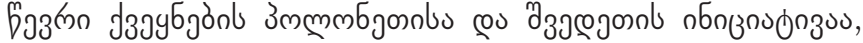

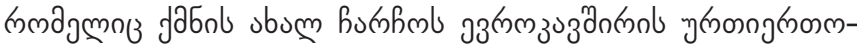

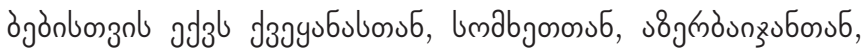

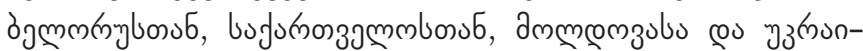

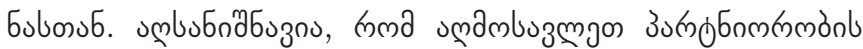

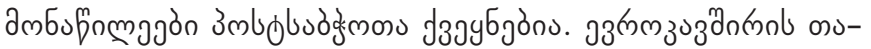

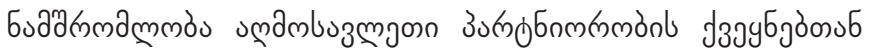

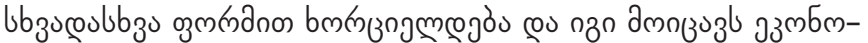

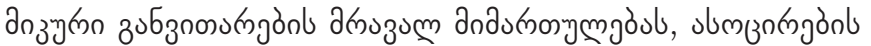

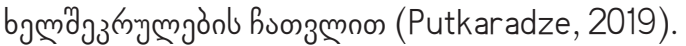

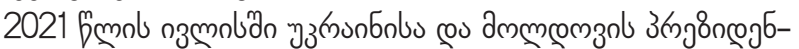

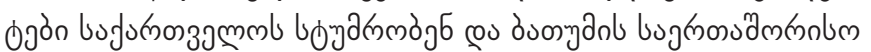

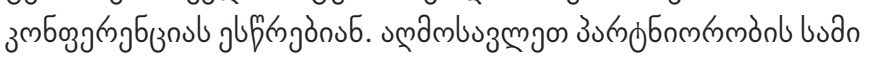

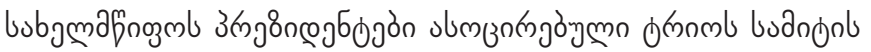

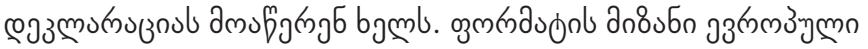

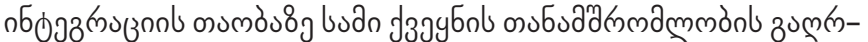

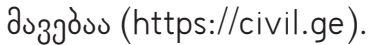

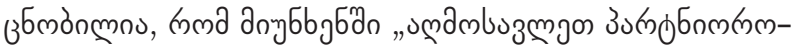

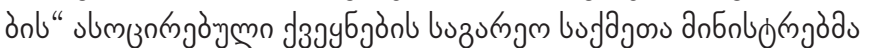

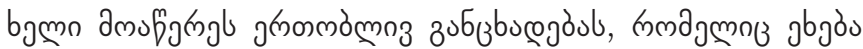

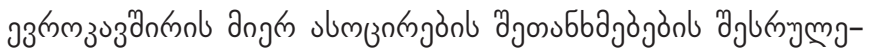

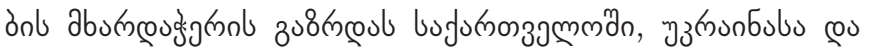

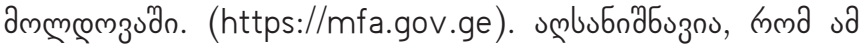

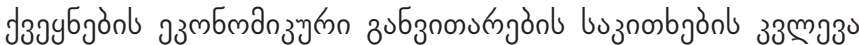

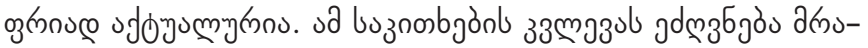

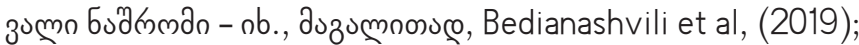
Papava, (2018); Gogolashvili, (2018); Putkaradze, (2010, 2019) pou bb3.

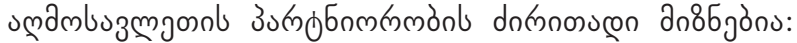

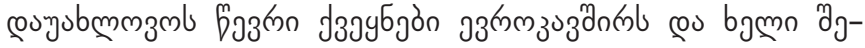
चf

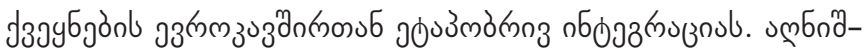

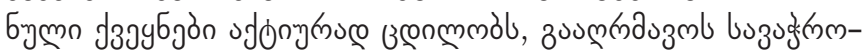

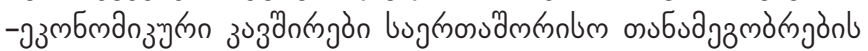

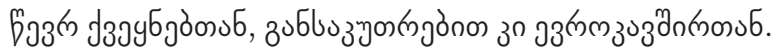

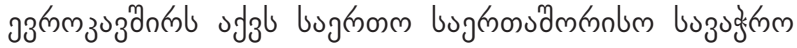

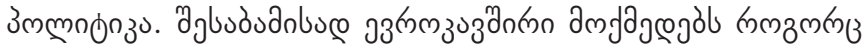

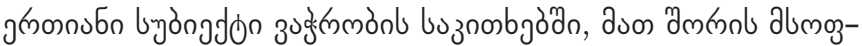

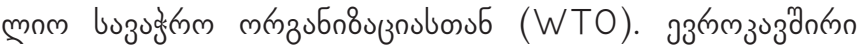

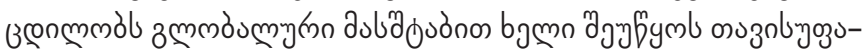

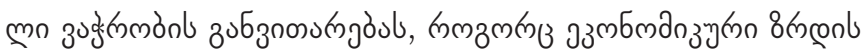

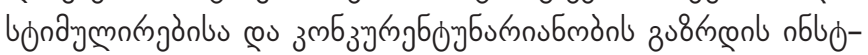

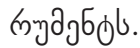

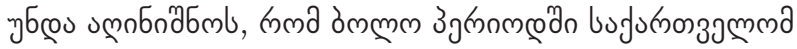

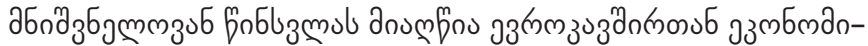

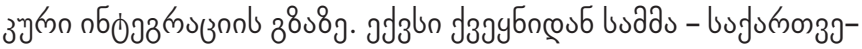

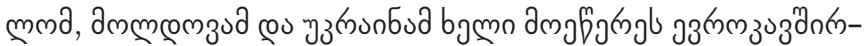

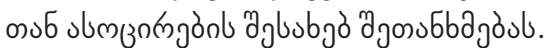

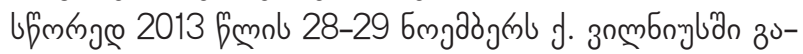

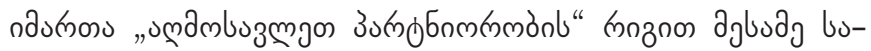

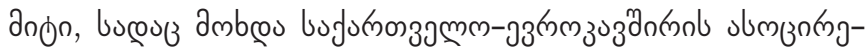

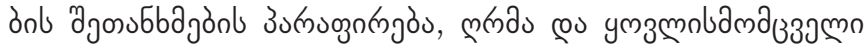

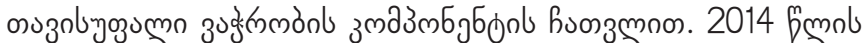

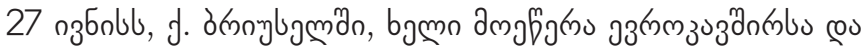

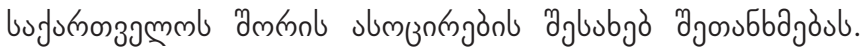

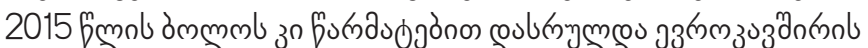
faz 


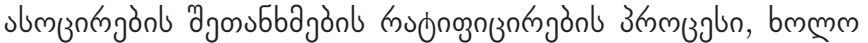

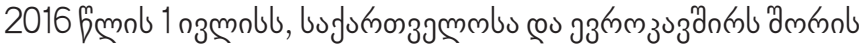

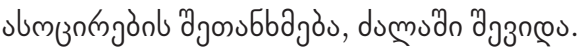

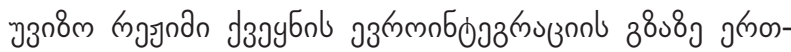
gknon चдбп

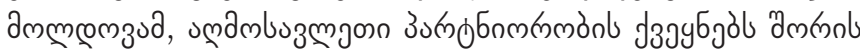

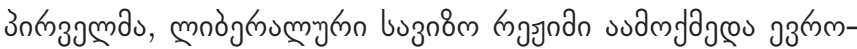

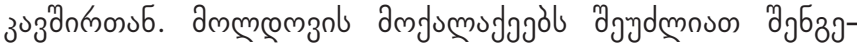

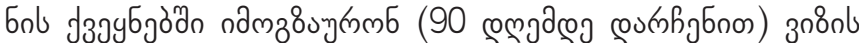

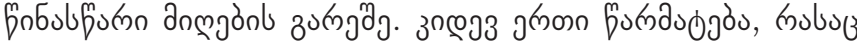

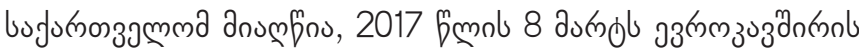

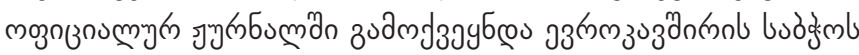

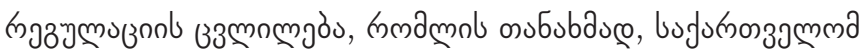

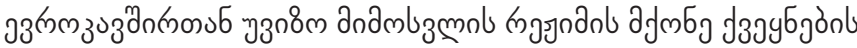

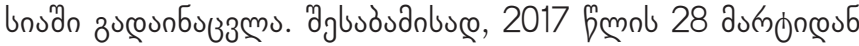

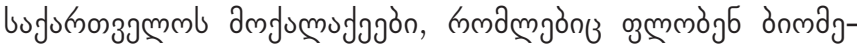

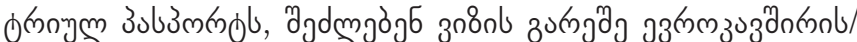

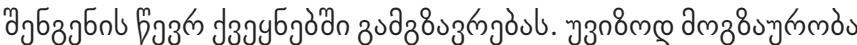

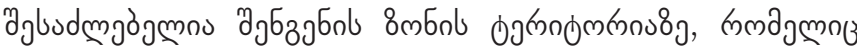

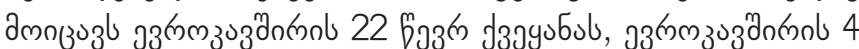

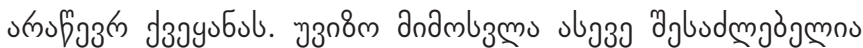
ә

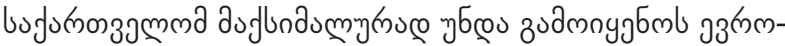

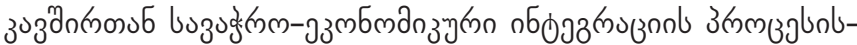

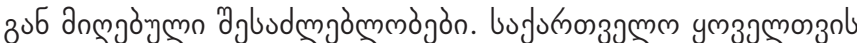

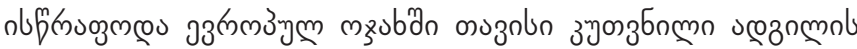
cosazзnюngon

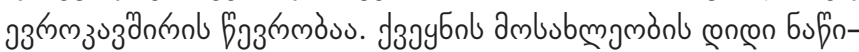

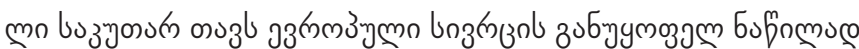

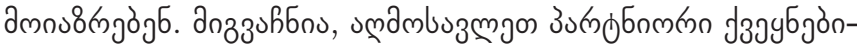

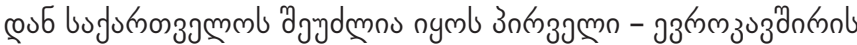

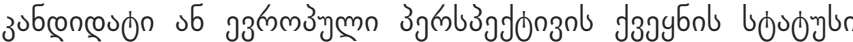
angbighmbl.

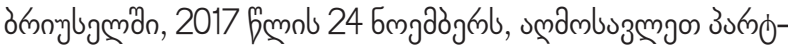
Enmknmònl ag-5 bsanon zundumons (European Commission,

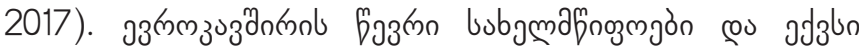

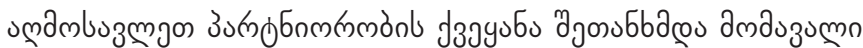

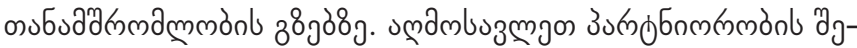

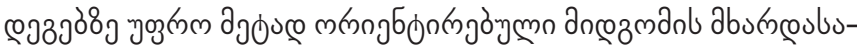

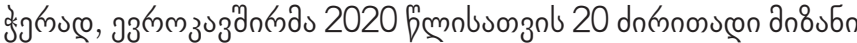

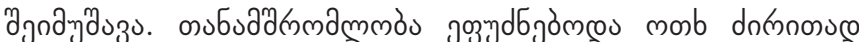

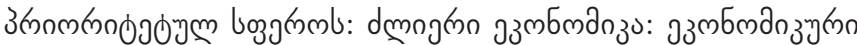

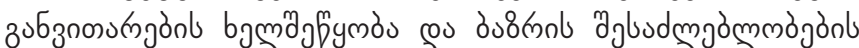
son

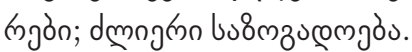

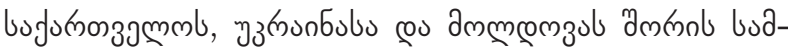

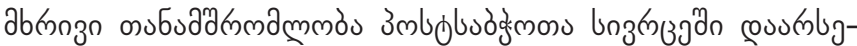

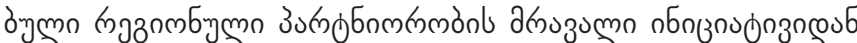

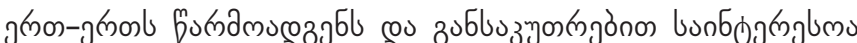

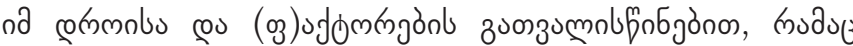

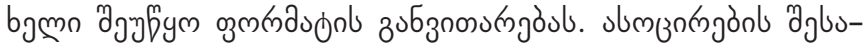

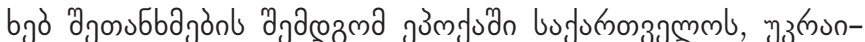

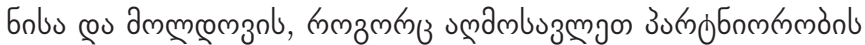

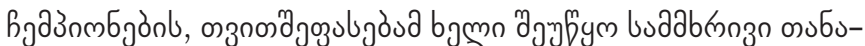

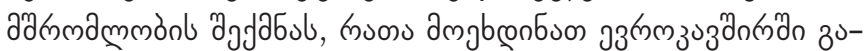

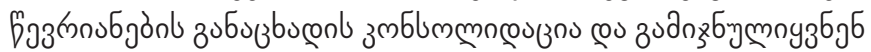

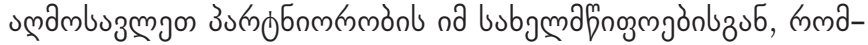

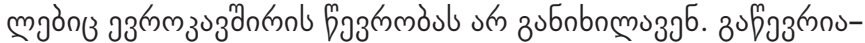

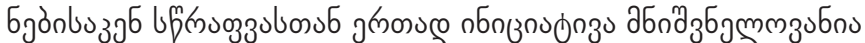

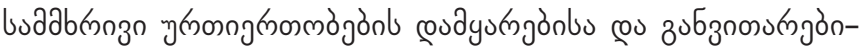

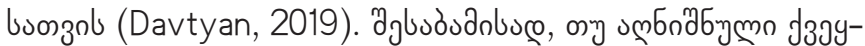
б бал з зu

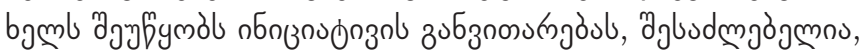

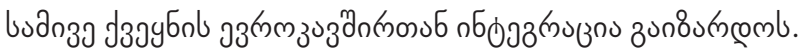

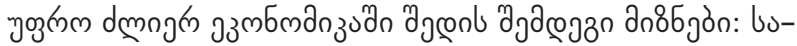

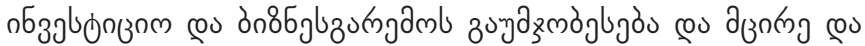

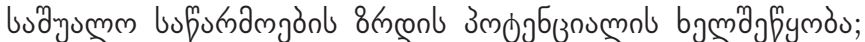

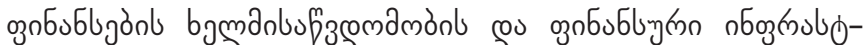

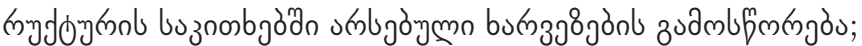

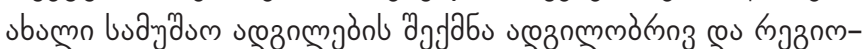

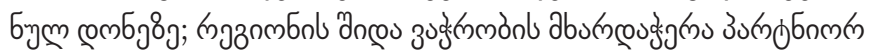

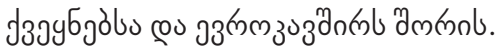

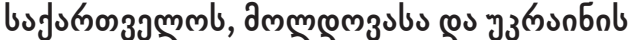

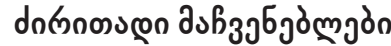

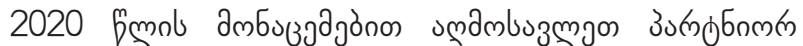

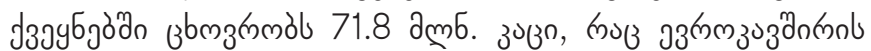
ambubmgmònb 16\%-l aju@gjbl (Eurostat, 2021), bmmm bs-

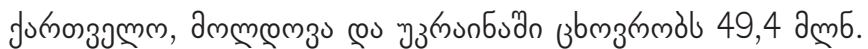

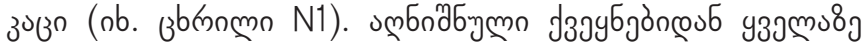

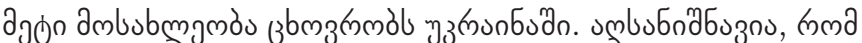

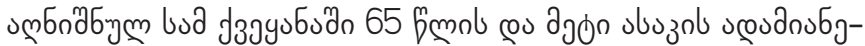

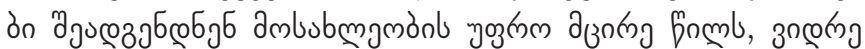

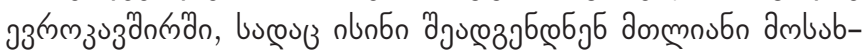

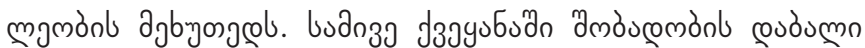

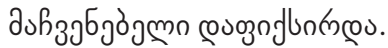

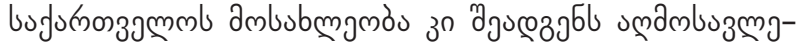

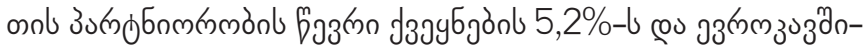

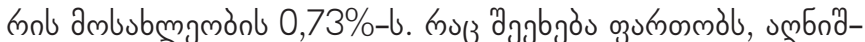

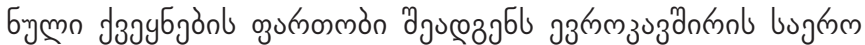

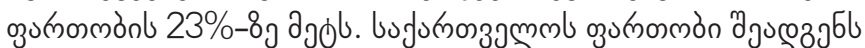

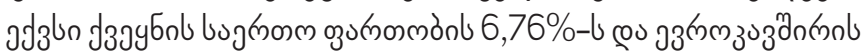
gुumonmònb 1,56\%-l.

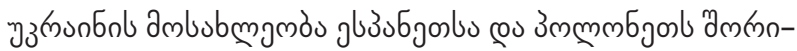

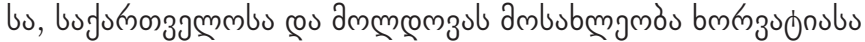

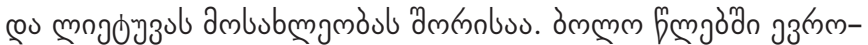

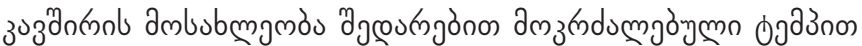

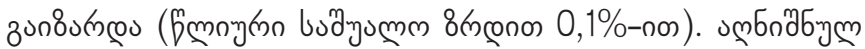




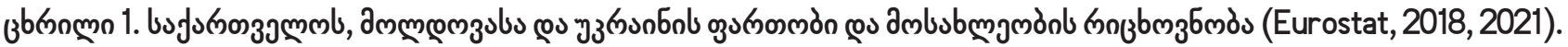

\begin{tabular}{|c|c|c|c|c|c|c|c|}
\hline & \multirow{2}{*}{ 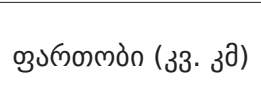 } & \multicolumn{6}{|c|}{ 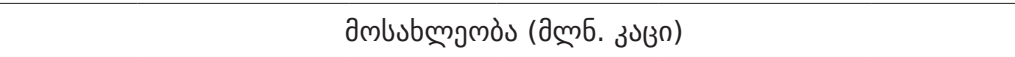 } \\
\hline & & 2009 & 2015 & 2017 & 2018 & 2019 & 2020 \\
\hline 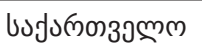 & 69700,0 & 4.4 & 3.7 & 3.7 & 3.7 & 3.7 & 3.7 \\
\hline дммूलмз & 33846,0 & 3.6 & 3.5 & 3.5 & 3.4 & 4 & 4 \\
\hline Уз๓๓о口бง & 603549,0 & 45.9 & 42.7 & 42.4 & 42.2 & 41.9 & 41.7 \\
\hline
\end{tabular}

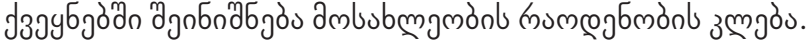

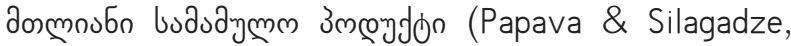

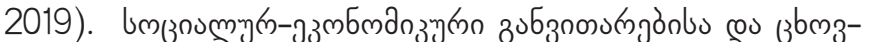

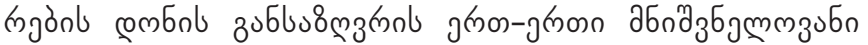

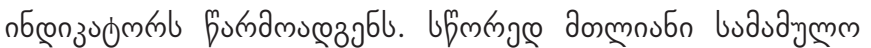

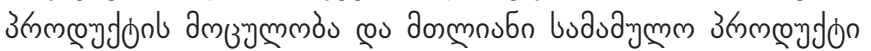

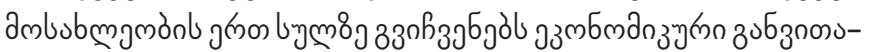

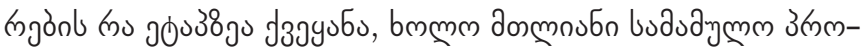

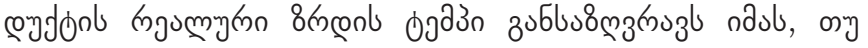

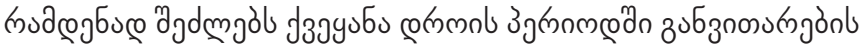

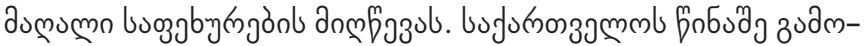

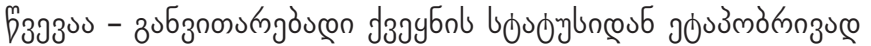

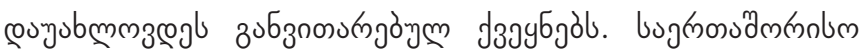

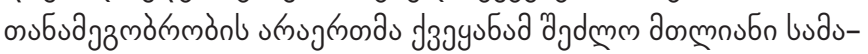

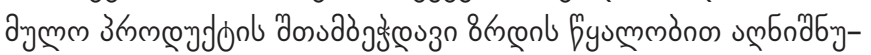

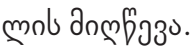

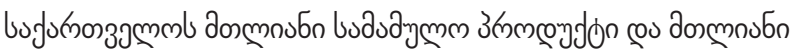

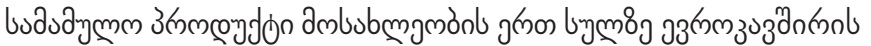

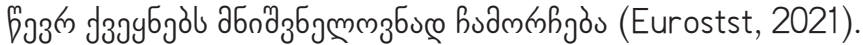

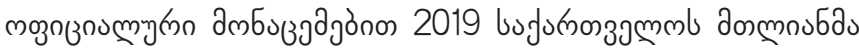

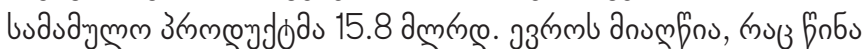

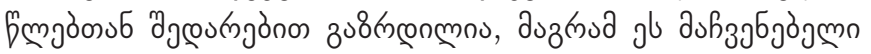

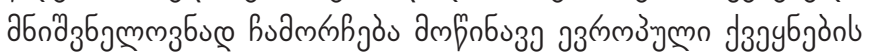

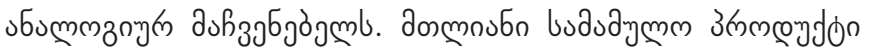

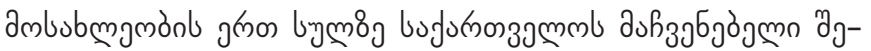
ง

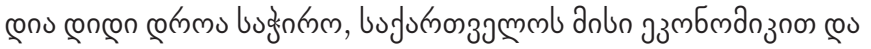

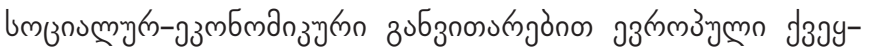

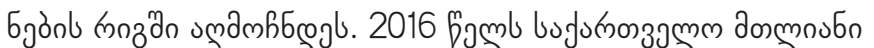

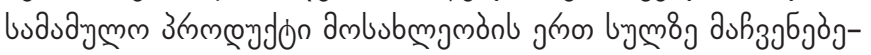

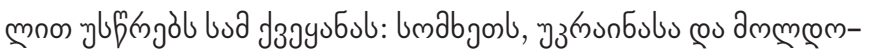

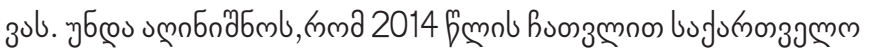

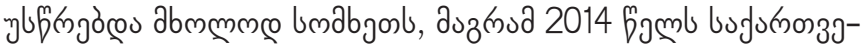

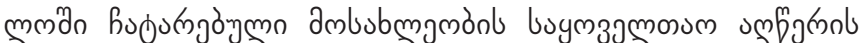

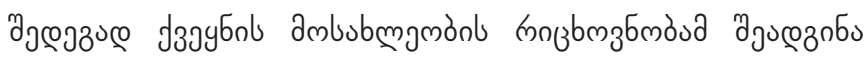

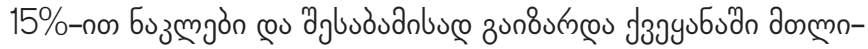

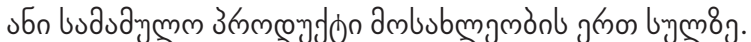

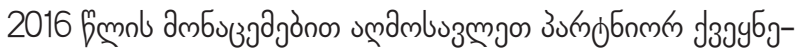

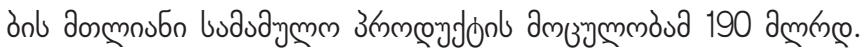

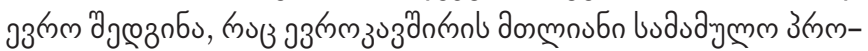

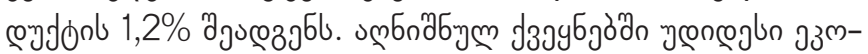

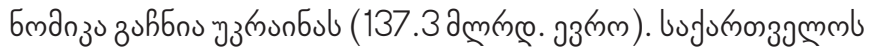

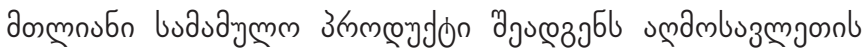

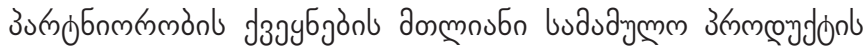

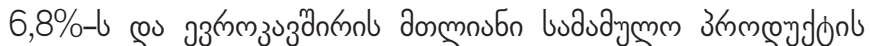
$0,1 \%-b$.

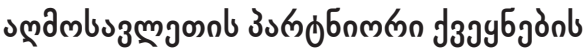

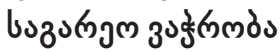

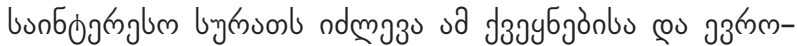
зu3

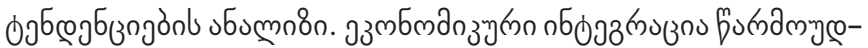

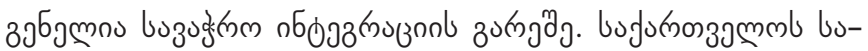

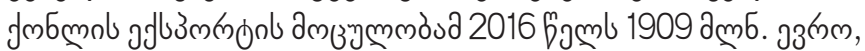

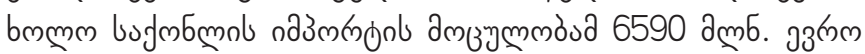

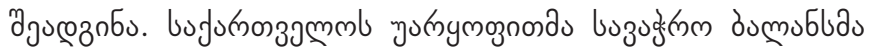

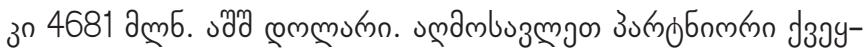

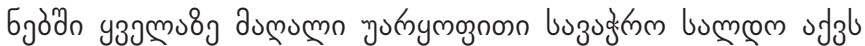

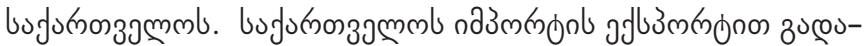

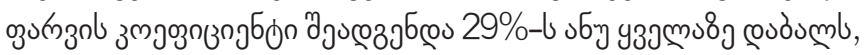

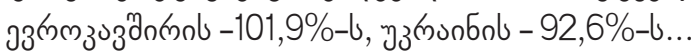

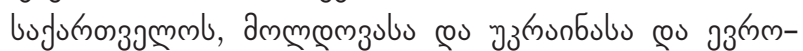

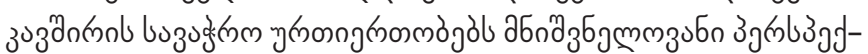

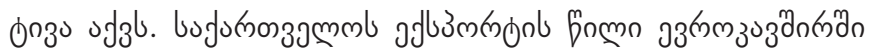

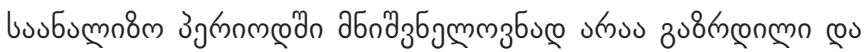

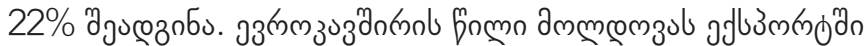

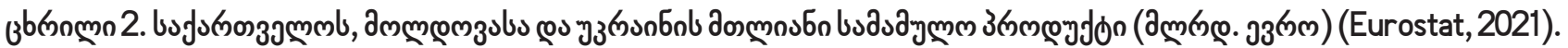

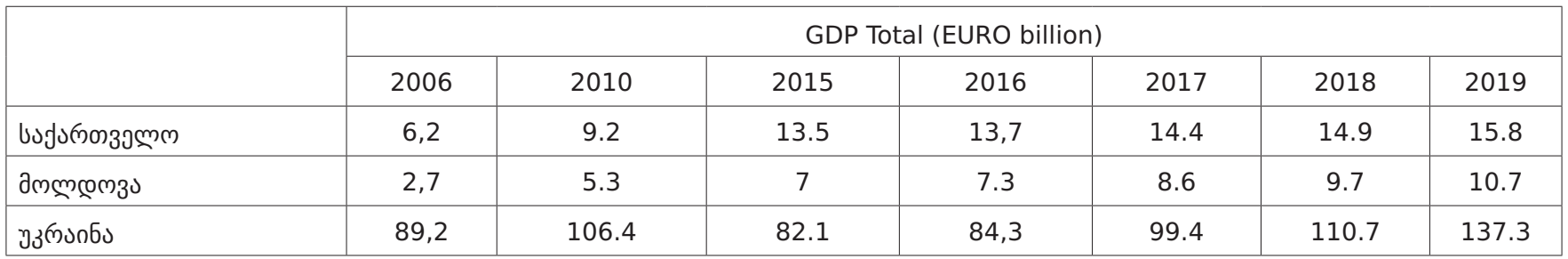




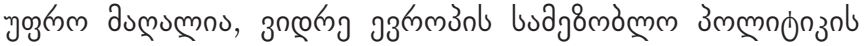

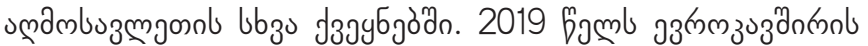

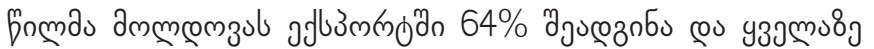

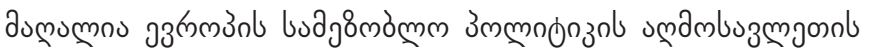

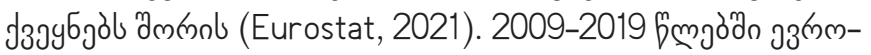

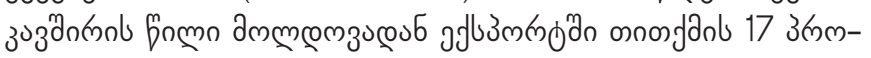

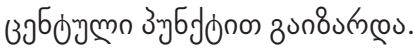

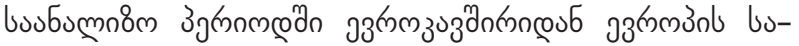

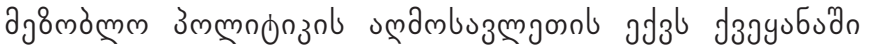

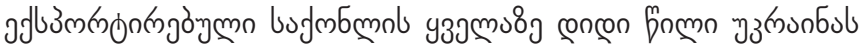

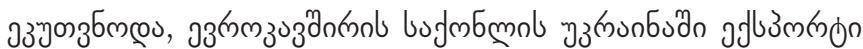

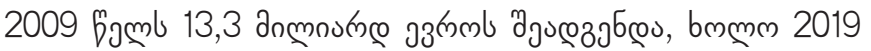

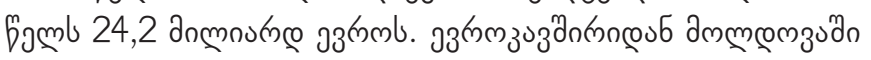

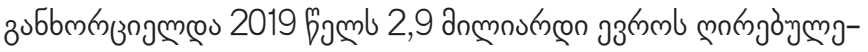

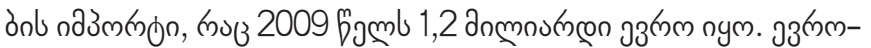

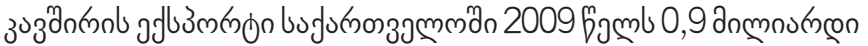

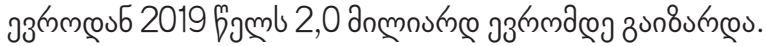

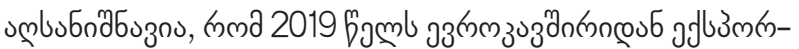

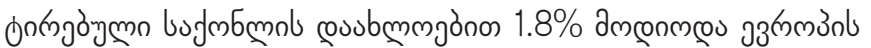

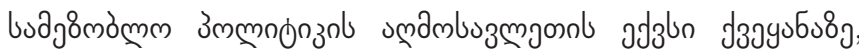

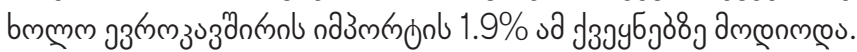

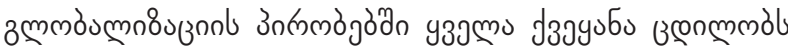

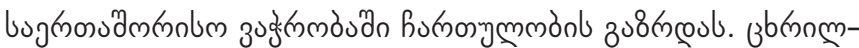

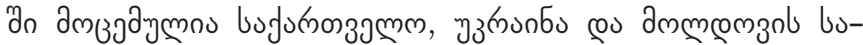

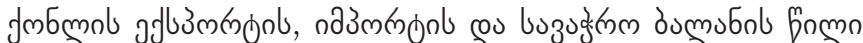

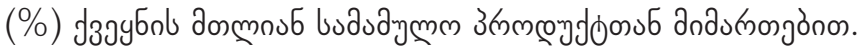

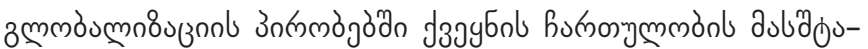

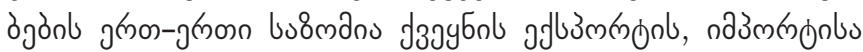

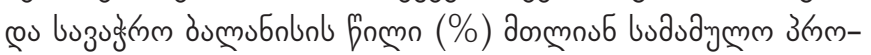

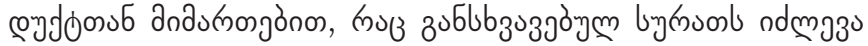

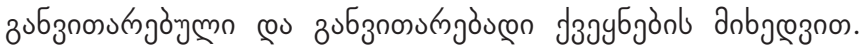

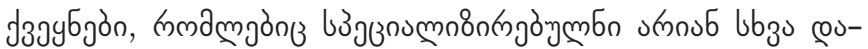

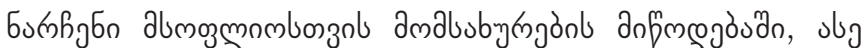

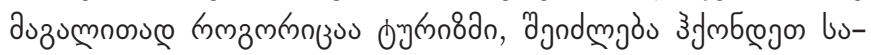

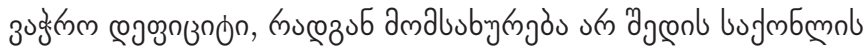

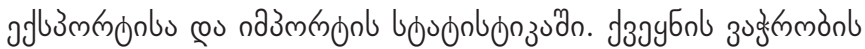

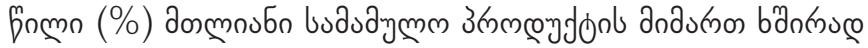

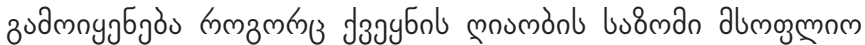

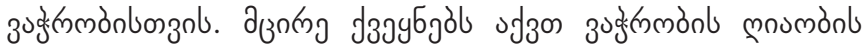

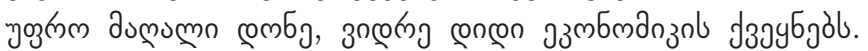

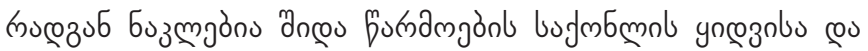

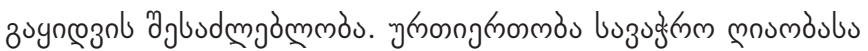

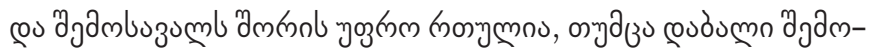

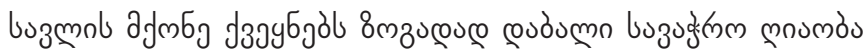
odzon.

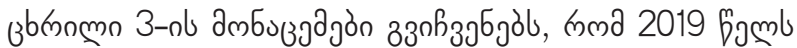

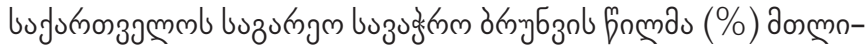

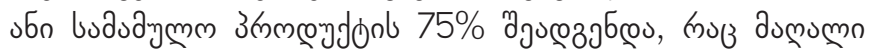
auf3jб

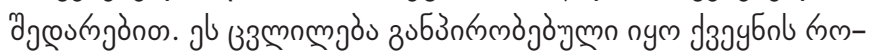

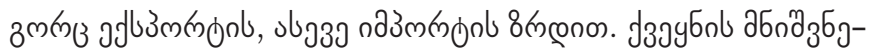

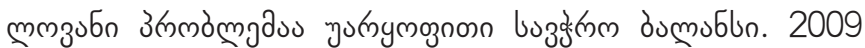

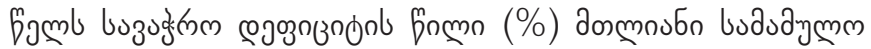

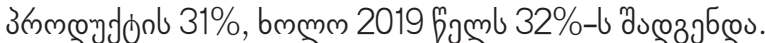

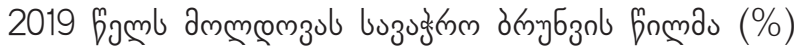

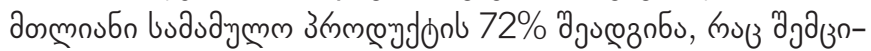

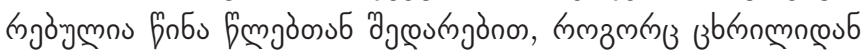

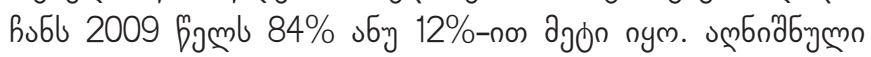

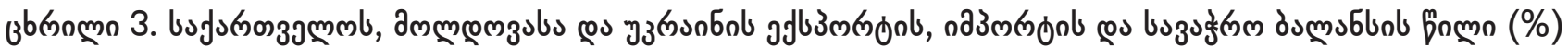

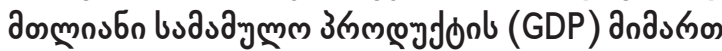

\begin{tabular}{|c|c|c|c|c|c|c|c|c|}
\hline & 2009 & 2010 & 2014 & 2015 & 2016 & 2017 & 2018 & 2019 \\
\hline \multicolumn{9}{|c|}{ 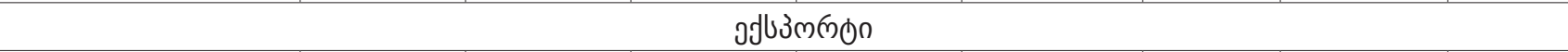 } \\
\hline 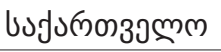 & 10.5 & 13.7 & 16.2 & 14.8 & 14.0 & 16.9 & 19.2 & 21.4 \\
\hline дммूкмз & 23.6 & 22.1 & 24.6 & 25.4 & 25.4 & 24.9 & 23.7 & 23.2 \\
\hline 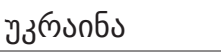 & 32.2 & 36.0 & 40.2 & 41.9 & 39.0 & 38.5 & 36.2 & 32.6 \\
\hline эз๓мзЈздипюп & 11.2 & 13.1 & 15.3 & 15.4 & 14.9 & 15.3 & 15.2 & 15.3 \\
\hline \multicolumn{9}{|c|}{ пазм๓ூ } \\
\hline 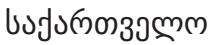 & 41.6 & 42.8 & 48.8 & 48.9 & 48.4 & 49.6 & 53.2 & 53.6 \\
\hline Әммкмз & 60.4 & 55.4 & 56.1 & 51.7 & 49.8 & 49.7 & 50.4 & 48.8 \\
\hline 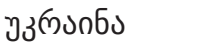 & 37.2 & 42.8 & 40.6 & 41.2 & 42.1 & 44.2 & 43.7 & 39.6 \\
\hline эз๓๓ззздипп & 11.3 & 13.4 & 13.8 & 13.5 & 12.8 & 13.6 & 14.1 & 13.9 \\
\hline \multicolumn{9}{|c|}{ 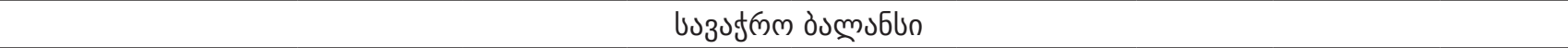 } \\
\hline 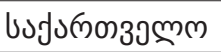 & -31.0 & -29.1 & -32.6 & -34.1 & -34.5 & -32.7 & -34.0 & -32.2 \\
\hline аммкомз & -36.8 & -33.4 & -31.5 & -26.2 & -24.5 & -24.8 & -26.7 & -25.6 \\
\hline 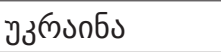 & -4.9 & -6.8 & -0.4 & 0.7 & -3.1 & -5.6 & 7.5 & 7.0 \\
\hline өзпмзјздпюп & -0.1 & -0.3 & 1.5 & 1.9 & 2.1 & 1.7 & 1.1 & 1.4 \\
\hline
\end{tabular}




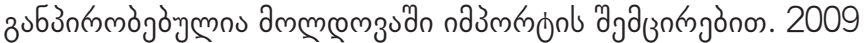

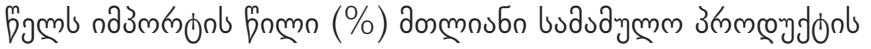

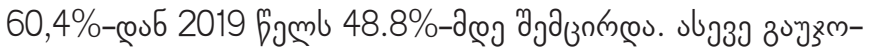

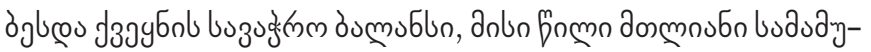

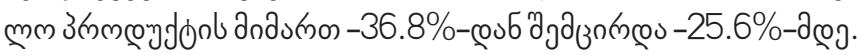

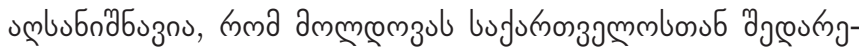

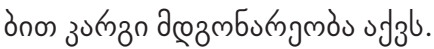

2019 fagmb चz

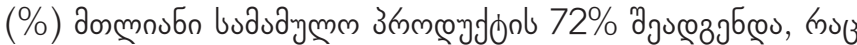

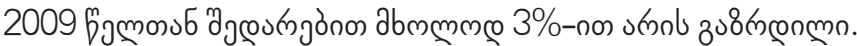

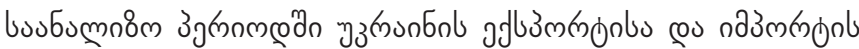

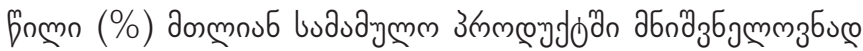
oknos do gusmanmo.

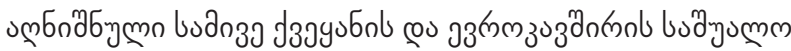

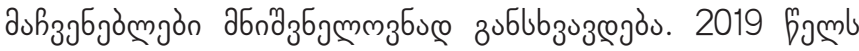

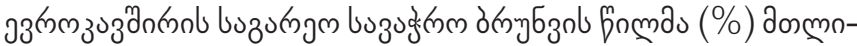

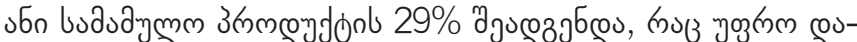

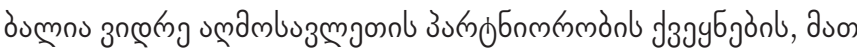

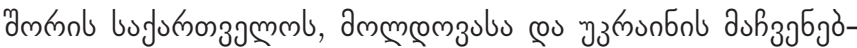

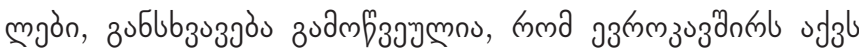

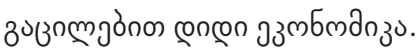

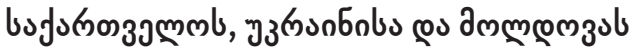

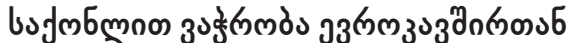

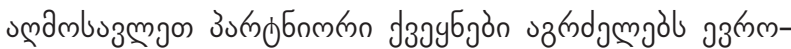

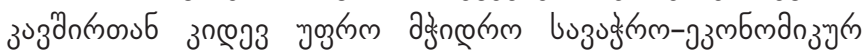

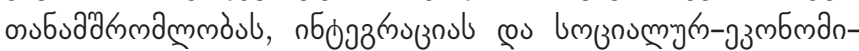

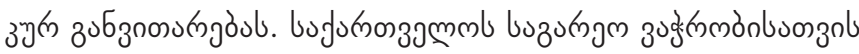

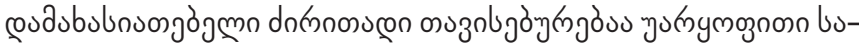

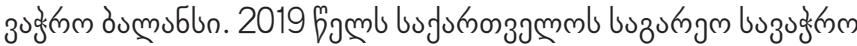

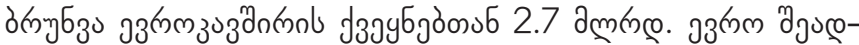

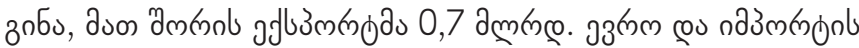

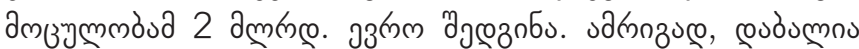

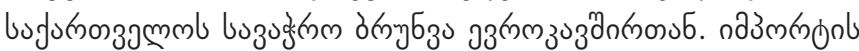

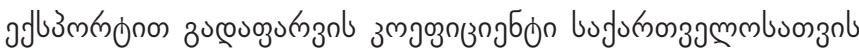

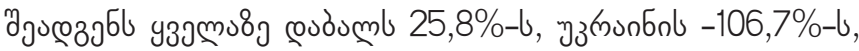
cos dmmengub - 67,6\%-b.

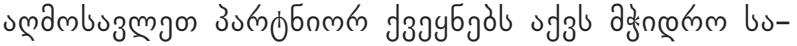

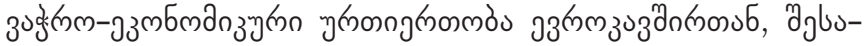

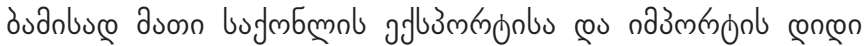

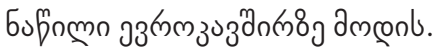

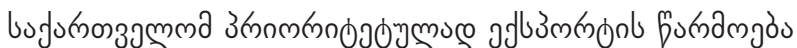

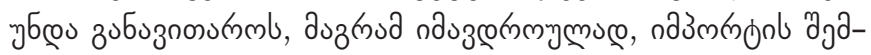

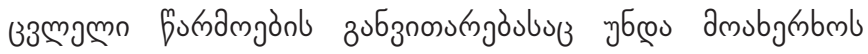
(Basilia T., Silagadze A., Chikvaidze T. (2001) 83. 429).

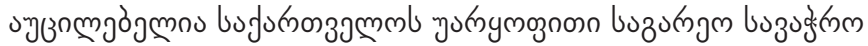

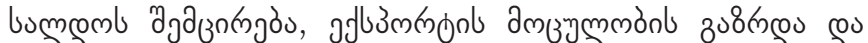

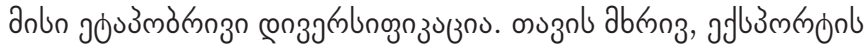

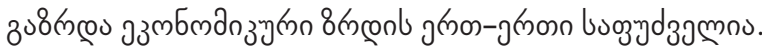

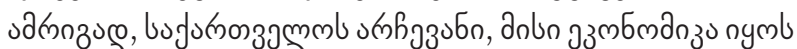
amfinбuз

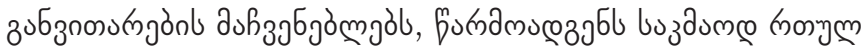

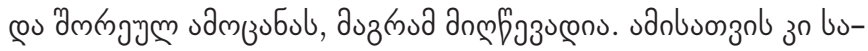

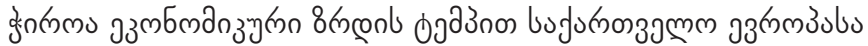

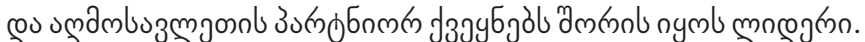

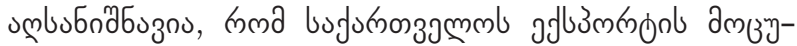

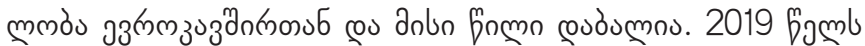

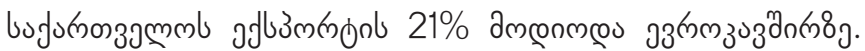

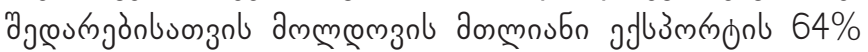

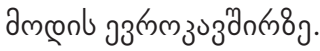

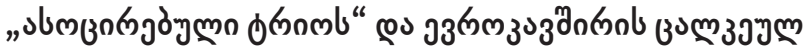

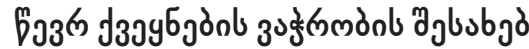

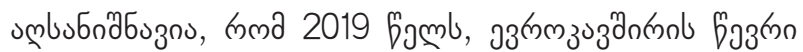

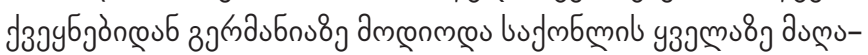

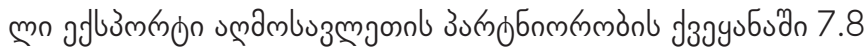

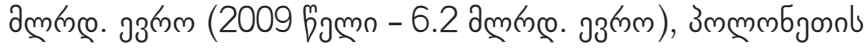

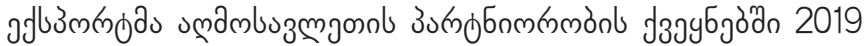

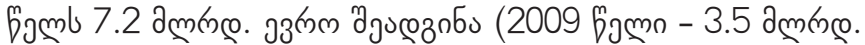

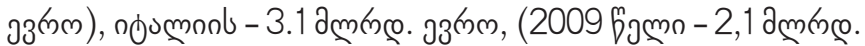

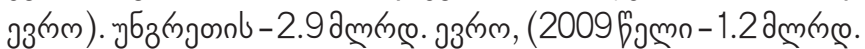

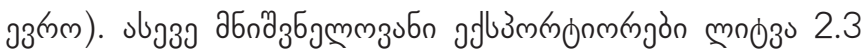

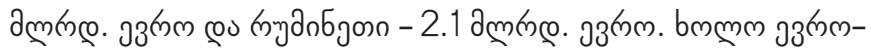

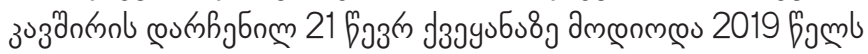

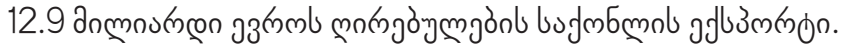

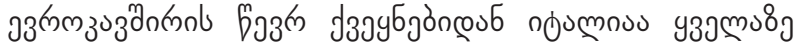

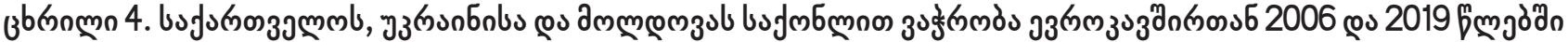

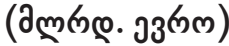

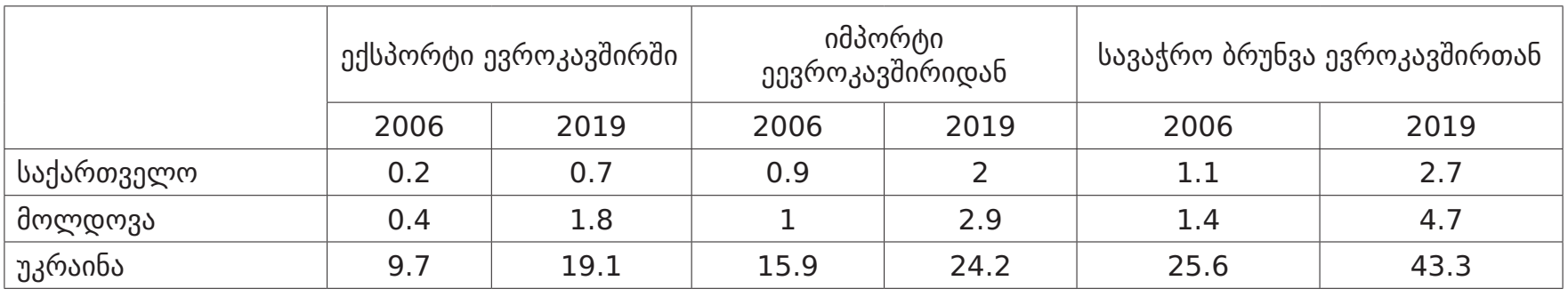




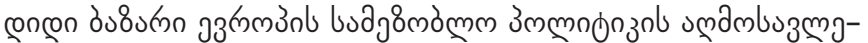

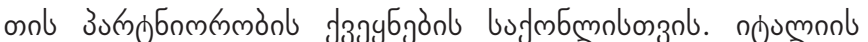

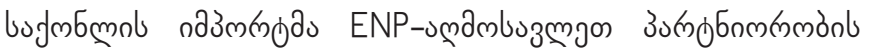

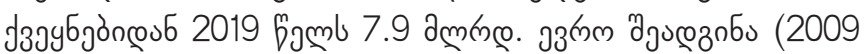

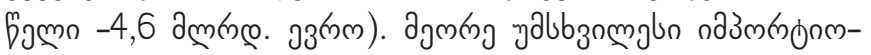

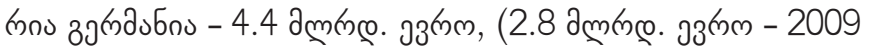

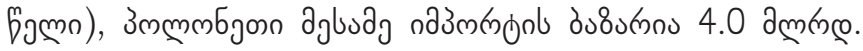

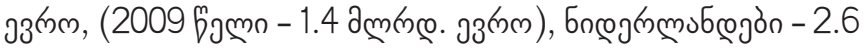

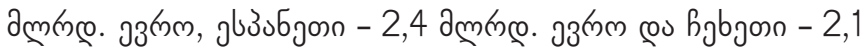

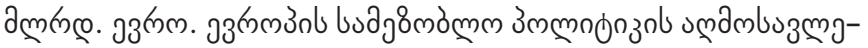

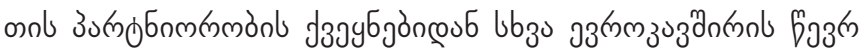

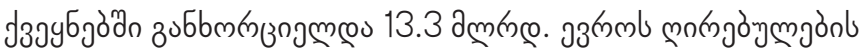

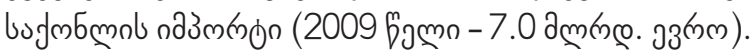

\section{@งเ3365000}

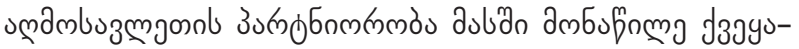

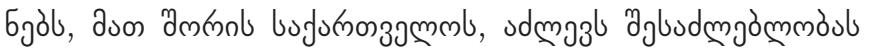

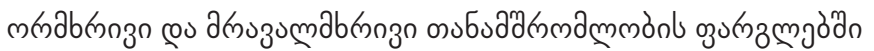

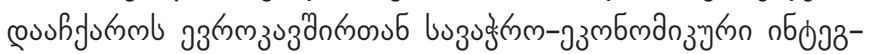

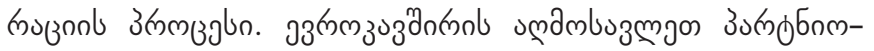

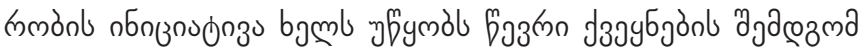

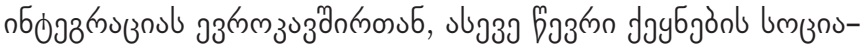

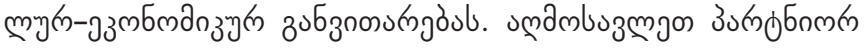

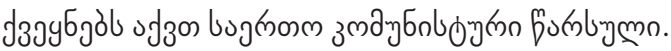

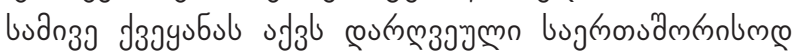

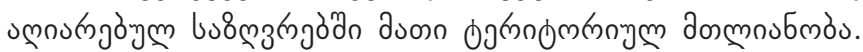

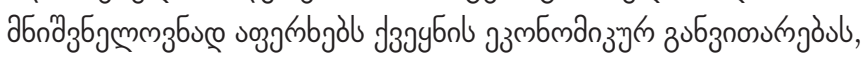

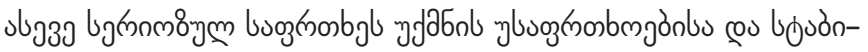

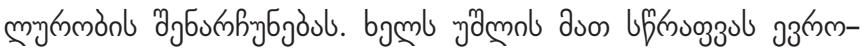

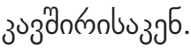

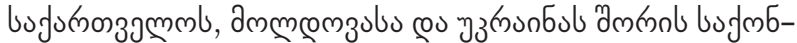

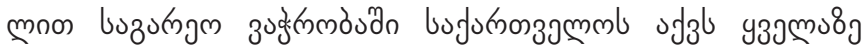

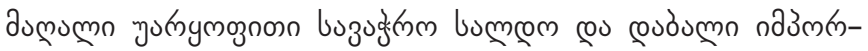

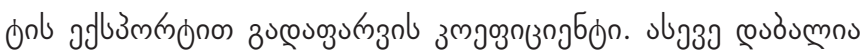

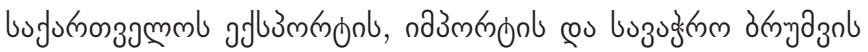

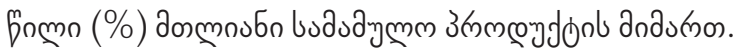

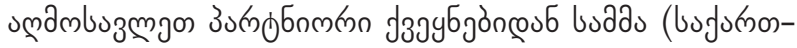

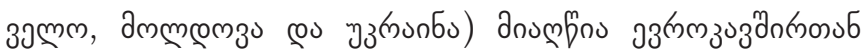

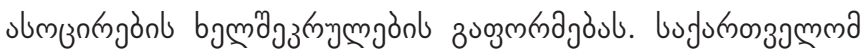

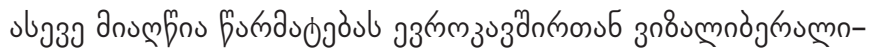
8uzanol żonbnon.

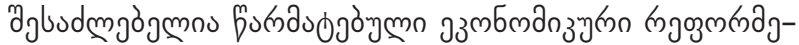

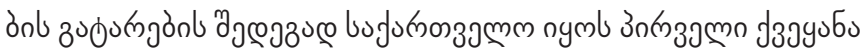

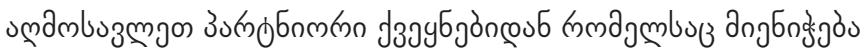

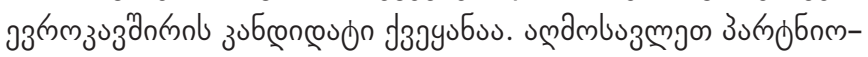

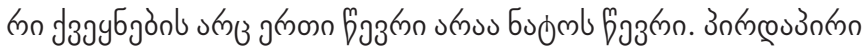

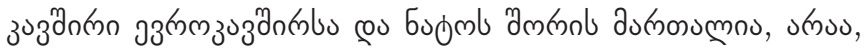

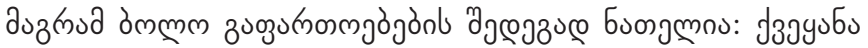

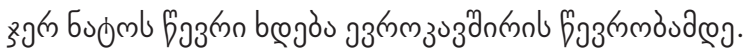

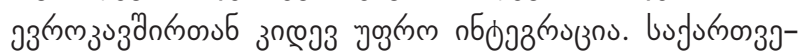

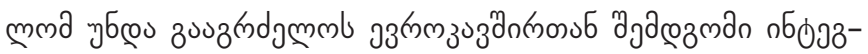

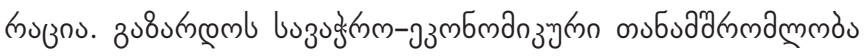

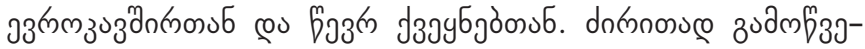

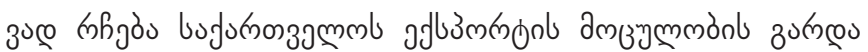

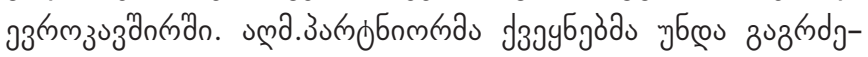

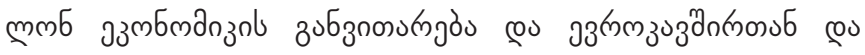

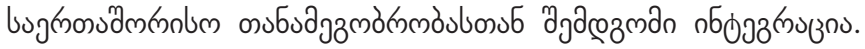

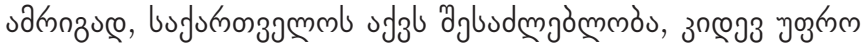

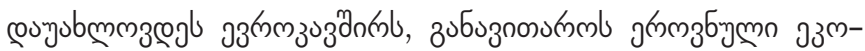

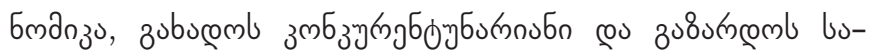

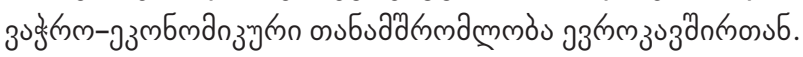

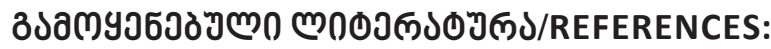

Basilia, T., Silagadze, A., \& Chikvaidze T. (2001) Post-Socialistic Transformation: Georgian Economy at the Threshold of the XXI Century. Tbilisi (in Georgian).

Bedianashvili, G. (2021). Macroeconomic and Cultural Determinants of the COVID-19 Pandemic Crisis. Bulletin of the Georgian National Academy of Sciences, 15 (2), 191-197.

Bedianashvili, G., Ivanov, Y., \& Paientko, T. (2019). Tax Reforms in Ukraine and Georgia: Changing Priorities. Journal of Tax Reform, 107-128.

Davtyan, E. (2019). Unpacking the Georgia-Ukraine-Moldova Cooperation: An Open-Ended or Narrowly Focused Partnership? Georgian Foundation for Strategic and International Studies.

Eteria, E. (2019). Georgia's Trade Performance in the Light of EU-Georgia Deep and Comprehencive Free Trade Area, Ecoforum Journal, 8(2/19).

European Commission. (2021). https://www.consilium.europa.eu/en/policies/eastern partnership/georgia/

Eurostat. (2018). Statistics on European Neighbourhood Policy Countries: East. Luxembourg: Publications Office of the European Union.

Eurostat. (2021). European Neighbourhood Policy countries - statistical overview. online Eurostat publication. 
Gogolashvili, K. (2018). Eastern Partnership Summit of 2017 and its Consequences. Rondeli Foundation. https://www.gfsis. org/ge/blog/view/771.

https://civil.ge/ka/archives/432958.

https://mfa.gov.ge. https://mfa.gov.ge/News/miunkhenshi-sami-asocirebuli-qveynis-saqartvelos,.aspx.

National Bureau of Statistics of the Republic of Moldova, www.statistica.md.

National Statistics Office of Georgia, www.geostat.ge.

Papava V., \& Silagadze A. (2019). "How the Term the "Gross Domestic Product" should be Translated into Georgian." Globalization and Business, 7,203-204. (In Georgian).

Papava, V. (2018) Assessment of Economic Growth in the Post-Communist Members of the European Union and the Eastern Partnership States. Georgian Foundation for Strategic and International Studies.

Putkaradze, R. (2012). Actual Issues of the Foreign Trade of Georgia. Paata Gugushvili Institute of Economics of Ivane JAvakhishvili Tbilisi State University. Proceedings of Materials of International Scientific-Practical Conference Dedicated to the 60th Birth Anniversary of Professor George Tsereteli National Economies and Globalization. 51-58. (In Georgian).

Putkaradze, R. (2015). Historical Aspects of Trade and Economic Relations between Georgia and the European Union. Zeszyty Naukowe Uniwersytetu Przyrodniczo-Humanistycznego W Siedlcach Seria: Administracjaizarządzanie. Scientific Journal, Nr 107, 2015. Zeszytynaukowe Uniwersytetu Przyrodniczo-Humanistycznego w Siedlcach Seria: AdministracjaiZarządzanie, Poland.

Putkaradze, R. (2019). Georgia in the European Union's Eastern Partnership Countries: Historical Aspects and Challenges. Journal, Ecoforum Journal. 8, Issue 1(18).

Putkaradze, R. (2010). Trade and Economic Relations between Georgia and the European Union: Problems and Perspectives. Universali, Tbilisi. (In Georgian).

Putkaradze, R., \& Chania, M. (2018). The Priorities of Georgian Economic Development in Conditions of Globalization. European Journal of Marketing and Economics, 1(1), 63-66.

Silagadze, A., \& Zubiashvili, T. (2015). Parameters of the European Union and the Post-Soviet Georgia's Economy. Refereed International Journal of Business and Management Studies (IJBMS).

State Statistics Service of Ukraine, ukrstat.org.

World bank (2019). World development indicators.

World Trade Organization (2020). World Trade Statistical Review. 